\title{
Greedy Cluster BASEd Routing For Wireless SENSOR NETWORKS
}

\author{
M. Parthasarathi ${ }^{1}$ and Dr. Karthikeyani Vajravel ${ }^{2}$ \\ ${ }^{1}$ Lecturer, Department of MCA, KSR College of Arts and Science, \\ Tiruchengode, Namakkal - Dt, India \\ ${ }^{2}$ Asst. Professor, Department of Computer science, \\ Thiruvalluvar Govt Arts college, Rasipuram, Namakkal Dt., India
}

\begin{abstract}
In recent years, applications of wireless sensor networks have evolved in many areas such as target tracking, environmental monitoring, military and medical applications. Wireless sensor network continuously collect and send data through sensor nodes from a specific region to a base station. But, data redundancy due to neighbouring sensors consumes energy, compromising the network lifetime. In order to improve the network lifetime, a novel cluster based local route search method, called, Greedy Clusterbased Routing (GCR) technique in wireless sensor network. The proposed GCR method uses arbitrary timer in order to participate cluster head selection process with maximum neighbour nodes and minimum distance between the source and base station. GCR constructs dynamic routing improving the rate of network lifetime through Mass Proportion value. Also, GCR uses a greedy route finding strategy for balancing energy consumption. Experimental results show that GCR achieves significant energy savings and prolong network lifetime.
\end{abstract}

\section{KEYWORDS}

Wireless Sensor Network, Cluster-based Routing, Greedy route, Cluster head, dynamic routing.

\section{INTRODUCTION}

In the recent past, several routing algorithms have been designed to improve the network lifetime in wireless sensor networks. In WSN, a multiple sensor nodes are distributed through the network. The data collected by the sensor nodes in WSN, are transmitted to a sink node. Therefore, the position of the sink node provides the great importance on the energy consumption and improving the lifetime of network. The mechanism adopted in clustering is highly significant in saving energy resources for network activities and has become promising area in the field of research.

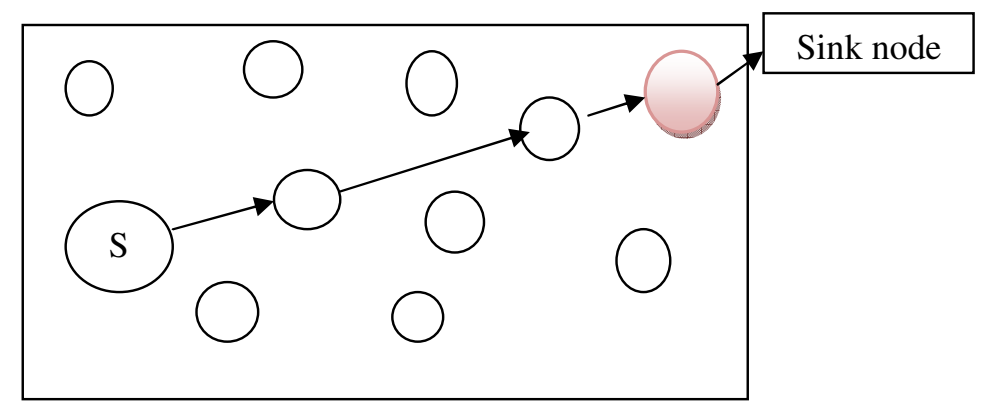

DOI: $10.5121 /$ ijcsit.2017.9208 
As shown in figure 1, the data collected by the source node are sent to sink node to forward the required destination node in WSN. Therefore the energy consumption and network lifetime is the major concern in the WSN.

Two-tier Particle Swarm Optimization for Clustering and Routing (TPSO-CR) [1] with a novel particle encoding scheme and fitness function identified the optimal routing tree to improve the packet delivery rate. However, network lifetime remained unaddressed. Energy Delay Index for Trade-off (EDIT) [2] was designed with the objective of improving the network lifetime with the help of Euclidean distance. However, the successful data delivery was not measured in terms of throughput. Another method to extend the network lifetime by using the fuzzy inference engine to select the super cluster head was presented in [3]. However, it increased the overhead.

Designing scalable routing in WSN with prolonged network lifetime is considered to be the most challenging task. In [4], a grid based method using upper Bollinger band and lower Bollinger band was designed aiming at improving the network lifetime. Clustering and multi-hop routing algorithms [5] was designed with the purview of improving network lifetime using inter-cluster and intra-cluster transmission tree.

Though many research works were conducted to improve network lifetime but at the cost of data being transmitted. To improve network lifetime and the data transmission rate, in [6], Rule Driven Multi-path Routing algorithm was designed for building cluster and transmitting data in an efficient manner.

In Wireless Sensor Networks (WSNs), one of the most influential factors next to the network lifetime is the energy efficiency. In [7], Energy Efficiency Semi Static Clustering (EESSC) based on Hierarchical Agglomerative Clustering (HAC) was designed to improve the WSN's energy efficiency. In [8], honey bee swarm intelligent based approach was designed to increase the amount of packets delivered to the base station.

An extended version including the fuzzy model was investigated in [9] for generating balanced clusters and prolonging the network lifetime was designed. Another Particle Swarm Optimization approach was presented in [10] to increase the total data packets to be delivered to the base station.

Energy conservation and fault tolerance plays major role in the deployment of a wireless sensor network (WSN). Therefore the design of clustering and routing algorithms should incorporate both these issues to prolong the network lifetime. In [11], a distributed clustering and routing algorithms jointly referred as Distributed Fault-tolerant Clustering and Routing (DFCR) was designed to prove the energy efficiency in case of fault tolerant. An integrated topology control and routing algorithm based on Mixed Integer Linear Programming model [12] was designed to optimally select the cluster head node.

Due to the limited energy possessed by the sensor nodes, increasing the network lifetime in WSN is a challenging task. This criticality increases with the increase in the density and topology change of network because of more data collections and packet transmissions. In [13], a clustering protocol based on Fan Shaped clustering was designed to improve both the energy saving and packet collection rate. In [14], adaptive clustering habit scheme was designed for wireless network to minimize the overall consumption of energy.

In [15], Clustering algorithm based on Ant Colony Optimization was investigated to manage inter-cluster and intra-cluster communication, varying the grid size of the network. In [16], cluster-based multi-path routing protocol was designed giving higher responsibility to the sink node for wireless sensor networks. A novel layer-based hybrid algorithm [17] for cluster head and 
cluster member selection was performed by way of information exchange between the neighbouring nodes until the cluster head node was selected.

Recent advances in miniaturization in the field of wireless communications have resulted in the making of micro sensors with limited processing and communicating capabilities. In [18], Energy Efficient Multilayered Architecture (EEMA) was designed to not only improve the network lifetime but also to reduce the routing delay. Another method based on Adaboost algorithm was investigated in [19] to minimize routing delay. Link cost function [20] was used to improve the packet delivery ratio and reduce energy consumption in WSN.

Based on the reviews, the existing methods has the limitations such as lack of network lifetime, reduced the delivery ratio and higher overhead. In order to overcome such kind of issues in WSN, a novel method is Greedy Cluster-based Routing (GCR) technique is introduced.

The contribution of the research paper is organized as follows; a novel Greedy Cluster-based Routing (GCR) technique is developed to extend the network lifetime in WSN. The GCR uses random timer for selecting the cluster head node. After that, Neighbour Node selection is carried out through Mass Proportion value for achieving the dynamic routing thereby improving network lifetime. Finally, GCR performs local route search using greedy strategy for selecting the neighbouring optimal node to forward data packet that is close to the destination node and it has sufficient energy for routing. This helps to reduce energy consumption during data forwarding.

The remainder of this paper is organized as follows. Section 2 provides an overview of Greedy Cluster-based Routing, with the aid of a network model. In Section 3, experimental results are presented and detailed discussion is included in Section 4. Finally, Section 5 concludes the work.

\section{Greedy Cluster-Based Routing For WSN}

In this section, an overview of the Greedy Cluster-based Routing (GCR) is presented starting with a design of network model to improve the routing efficiency in terms of network lifetime, reducing the energy consumption during data forwarding.

\subsection{Network model}

Let us consider a wireless network with number of sensor nodes that are uniformly deployed in ' $m * m$ ' square area represented as a simple digraph ' $G=(V, E)$ ' with transmission range ' $R$ '. The network consists of ' $n$ ' wireless sensor nodes represented as ' $V=\left\{v_{1}, v_{2}, \ldots, v_{n}\right\}$ ' and ' $E=\left\{e_{1}, e_{2}, \ldots, e_{n}\right\}$ ' representing the set of communication links between the sensor nodes. Suppose an adjacent pair ' $v_{j}, v_{k}$ ', then ' $e_{i}\left(v_{j}, v_{k}\right)$ ' represents that both sensor nodes ' $v_{j}$ and ' $v_{k}$ ' lie within the transmission range ' $R$ ' and is expressed as given below.

$$
E=\left\{e_{i}\left(v_{j}, v_{k}\right) \mid \operatorname{Dis}\left(v_{j}, v_{k}\right) \leq R\right\} \text {, where } v_{j}, v_{k} \in V
$$

\subsection{Problem definition}

The cluster head node in wireless network based on the design of cluster consumes more energy during intra-cluster and inter-cluster communication. The energy consumption during intracluster communication is highly influenced by the cluster size, where the energy consumption increases with number of sensor nodes in cluster. As the sensor node in the wireless sensor networks has limited transmission range, multi-hop design is used for data transmission. During data transmission phase, cluster heads act as intermediate nodes to distribute data between source and destination in WSN. During the inter-cluster communication, the cluster heads nearer to the 
neighbourhood of base station overcomes heavy traffic and expires faster than far away cluster heads. This process improves the control overhead. In order to overcome such kind of issues a Greedy Cluster Based Routing Protocol is designed.

\subsection{Greedy Cluster-based Routing}

The design of Greedy Cluster-based Routing (GCR) method is carried out aiming at improving the network lifetime in WSN. Specifically, there are three parameters such as energy consumption, network lifetime and throughput are considerably affects the network performance. Initially, the Cluster Based Routing Protocol is applied to improve the network lifetime through the arbitrary timer. Secondly, Mass Proportion-based Neighbour Node selection measure is performed for selecting the neighbouring node improving the throughput. Finally, Greedy Local route search algorithm used for reducing the energy consumption in WSN. The architecture diagram of the GCR) method is shown in figure 1.

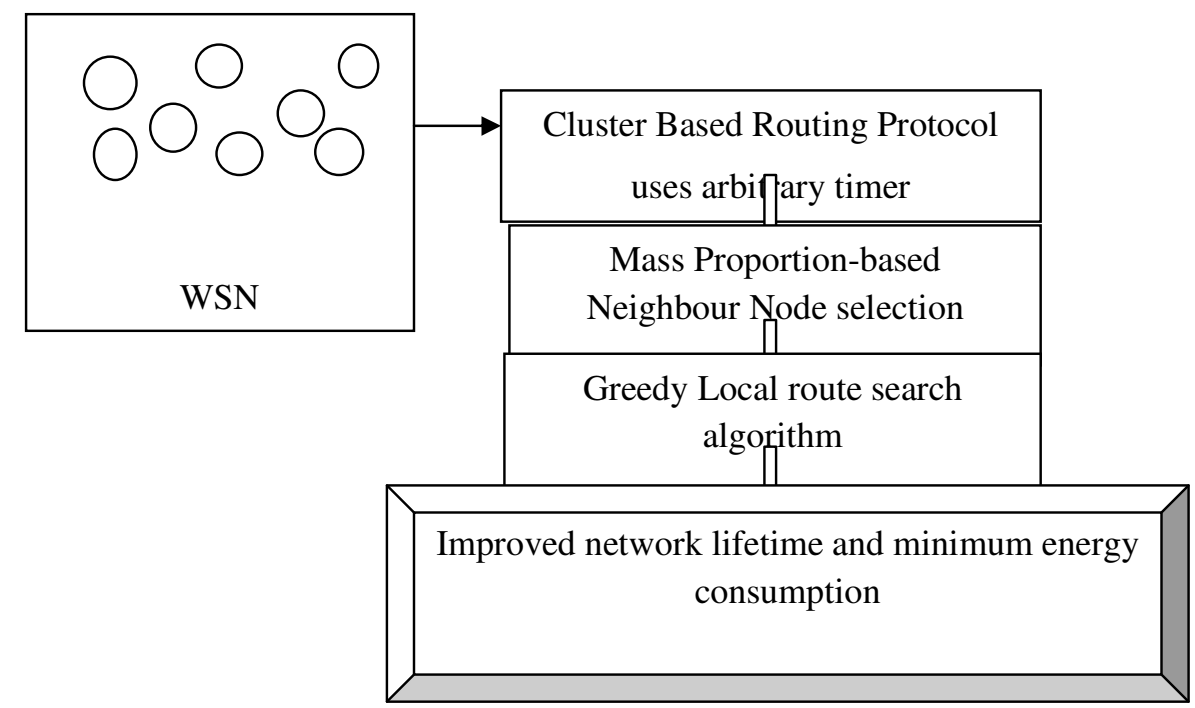

Figure 2 Architecture diagram of Greedy Cluster-based Routing (GCR) method

Figure 2 shows the architecture diagram of the Greedy Cluster-based Routing (GCR) method. The GCR method performs efficient transmission and improves the network lifetime in order to obtain the higher throughput and reduce the energy consumption in WSN. The description about GCR method is explained in below two subsections.

\subsection{Cluster Based Routing Protocol}

The Cluster Based Routing Protocol (CBRP) in the GCR uses an arbitary timer during data packets forwarding to minimize the control overhead via the location information of the sensor nodes in the network. The CBRP uses random timer for selecting the cluster head node. During network initialization stage, the base station broadcasts a beacon message to all the sensor nodes in the network. To find the neighbour nodes, the GCR uses maximum mass proportion value which is discussed in the next section. During data packets forwarding, each node initiates a timer and is expressed as given below.

$$
\gamma=\sum_{i=1}^{n} \frac{\operatorname{Dis}(S N, B S)}{N N_{i}}
$$




$$
T=\frac{\beta}{\gamma}
$$

From (2), 'Dis (SN,BS)' corresponds to the distance between the source node ' $S N$ ' and the base station ' $B S$ ' with a total of ' $N N_{i}$ ' neighbouring nodes. From (3), ' $T$ ' represents the timer whereas ' $\beta$ ' corresponds to the timer value between ' $[0,1]$ '. With the aid of ' $\gamma$ ', each sensor node has more neighbouring nodes with minimum distance to the base station is identified. Figure 1 shows the construction of cluster based routing protocol.
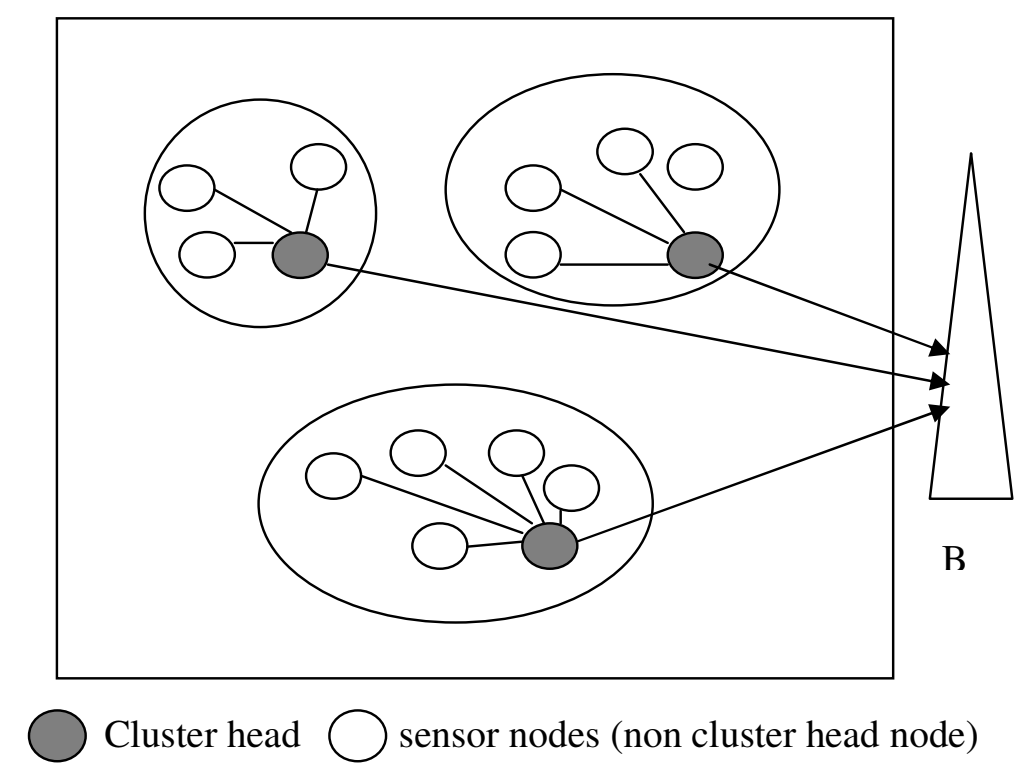

Figure 3 Cluster Based Routing Protocol

The sensor node completes the timer ' $T$ ' is the cluster head ' $\mathrm{CH}$ ', node for that cluster. As shown in the figure 3, the gray circle completes the timer forms the cluster head node. The remaining sensor node enters the cluster by sending a join message to the cluster head node and is expressed as given below.

$$
\sum_{i=1}^{b} S_{i}=\sum_{i=1}^{n} C[\operatorname{Join}[\mathrm{CH}]]
$$

Once the cluster formation is accomplished then the each sensor nodes measures the arbitrary timer in order to participate in the cluster head selection process. The arbitrary timer ' $A T$ ' is expressed as given below.

$$
\begin{aligned}
& X=E_{t} * E_{u} \\
& A T=\sum_{i=1}^{n}\left(1-\frac{X}{N N_{i}}\right)
\end{aligned}
$$

From (6), the arbitrary timer ' $A T$ ' is obtained using the total energy ' $E_{t}$ ', ' $E_{u}$ ', the utilized energy and the remaining neighbouring nodes ' $N N_{i}$ ' respectively. Figure 2 shows the cluster based routing algorithm.

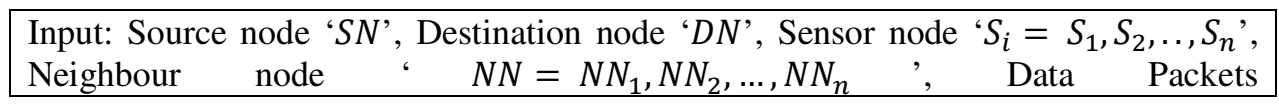




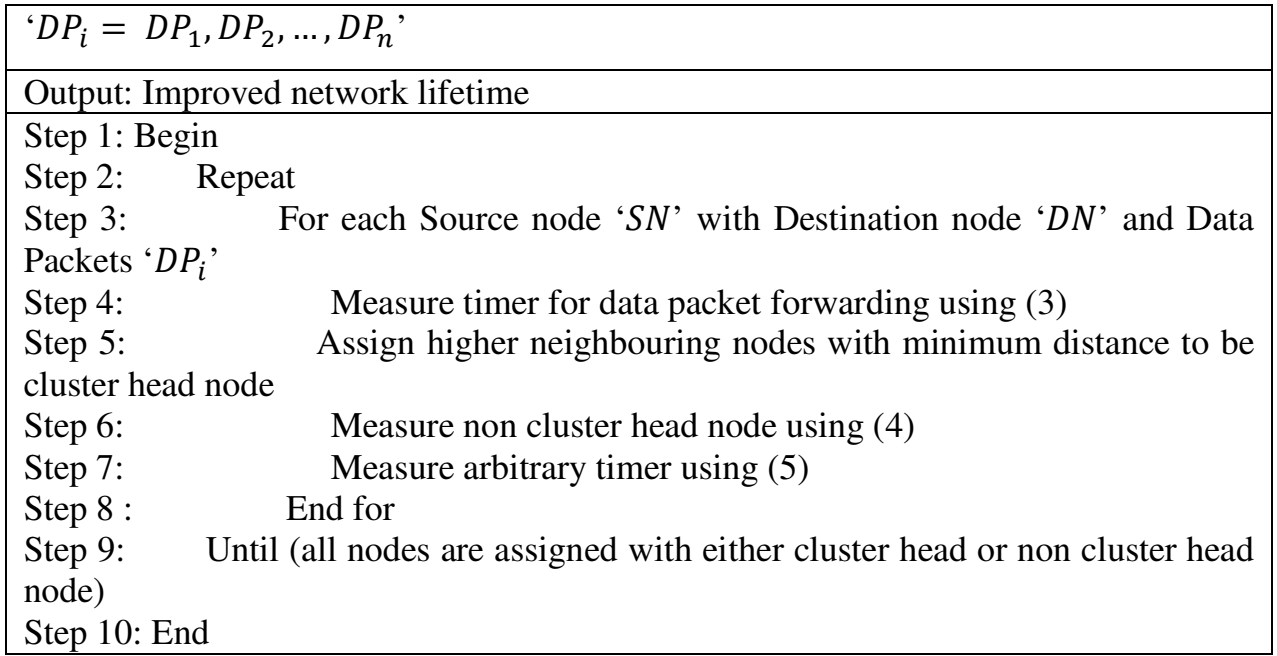

Figure 4 Cluster based routing algorithm

As shown in figure 4, each sensor node starts its timer using its local information to participate in the cluster head selection process. During cluster head selection process, sensor nodes uses energy consumption cost ' $X$ ' as the influential factor. The neighbourhood node selection and the total remaining neighbouring nodes play an important role in selecting a cluster head for the specific cluster. The neighbourhood node selection is performed by applying mass proportion value that is discussed in the next section. The GCR uses only local information to select cluster heads and reducing the control overhead. This helps to improve the network lifetime.

\subsection{Mass Proportion-based Neighbour Node selection}

The GCR employed for neighbour node selection based on the mass proportion value. Let us assume that each sensor node initially has single sensed information (i.e. data packets) to be sent to the base station in the network and each sensor node attracts its neighbouring nodes with mass proportion value. Figure 4 shows the Neighbour Node selection based on Mass Proportion value.

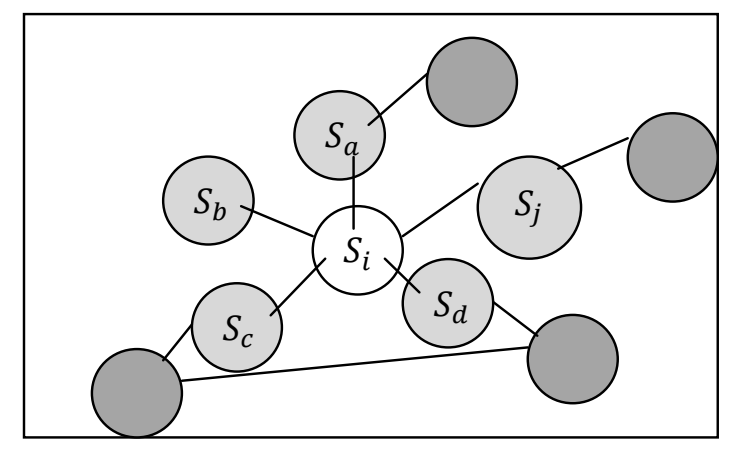

Figure 5 Neighbour node selections

Let us consider the sensor nodes ' $S_{i}$ ' and ' $S_{j}$ ' in the figure 3 . As shown in the figure 5 , the neighbour nodes of ' $S_{i}$ ' is selected within one hop away from node ' $S_{i}$ '. Here, the gray circles are the neighbours of sensor node ' $S_{i}$ '. On the other hand, dark gray circles form the neighbours of the neighbouring node ' $S_{i}$ '. After that, the mass proportion value between sensor node ' $S_{i}$ ' and 
' $S_{j}$ ', ' $M P_{i j}$ ' is directly proportional to the mass of the sensor node and the mass of the neighbouring sensor nodes and inversely proportional to the square of the distance between them. The mass proportion value is as expressed below.

$$
M P_{i j}=l \frac{M_{i} * M_{j}}{\operatorname{Dis}\left(S_{i}, S_{j}\right)^{2}}
$$

From (7), ' $l$ ' symbolizes the constant, ' $M_{i}$ ' and ' $M_{j}$ ' represents the mass of the sensor node ' $S_{i}$ ' and ' $S_{j}$ ' with the distance represented by ' $\operatorname{Dis}\left(S_{i}, S_{j}\right)$ ' respectively. Figure 3 illustrates the basic idea of neighbour node selection applied in the proposed GCR method. The sensor node ' $S_{i}$ ' is attracted by all its neighbours in different directions and the mass value for all the neighbouring sensor nodes are different. Therefore, the major concern is in selecting the neighbour of the ' $S_{i}$ ' node.

In the proposed method, the neighbour with the maximum mass proportion value is selected as the next neighbouring node and is expressed as given below.

$$
\sum_{i=1}^{b} S_{i} \rightarrow N N_{i}=\operatorname{MAX}\left(M P_{i j}\right)
$$

Due to the diversified nature of the network and the changing topology, the energy of each sensor node varies and their mass proportion value also varied. In this way, dynamic routing is achieved by measuring different mass proportion value for several sensor nodes thereby improving the rate of network lifetime.

\subsection{Local route search algorithm}

Finally, a local route search algorithm called greedy forwarding strategy is designed with the obtained neighbouring nodes through which the data packets are transmitted. The local route search algorithm based on greedy forwarding uses local information with the objective of brining the data packets closer to the destination.

As the GCR applies greedy strategy for local route search between the source and destination, a function that describes which neighbouring nodes is the most optimal node. Obviously, the GCR selects the optimal node to forward data packet which is both close to the destination node and has enough energy for routing. This is obtained by the expression given below.

$$
f=\sum_{i=1}^{n} N N_{i}\left(M I N\left[\left(E_{1}, E_{2}, \ldots, E_{j}\right)\right] U\left[\operatorname{Dis}\left(S_{i}, B S\right)\right]\right)
$$

The Greedy Strategy-based Local Route Search algorithm is given in figure 4.

\begin{tabular}{l} 
Input: Source node ' $S N$ ', Destination node ' $D N$ ', Sensor node ' $S_{i}=S_{1}, S_{2}, \ldots, S_{n}$ ', \\
Neighbour node ' $N N=N N_{1}, N N_{2}, \ldots, N N_{n}$ ', Data Packets ' $D P_{i}=D P_{1}, D P_{2}, \ldots, D P_{n}$ ', \\
Base Station ' $B S$ ' \\
\hline Output :Optimal energy consumption \\
\hline Begin \\
For each Source node ' $S N$ ' with Destination node ' $D N$ ' and Data Packets ' $D P_{i}$ ' \\
For sensor nodes ' $S_{i}$ ' and ' $S_{j}$ ' \\
Measure the mass proportion value using (7) \\
Obtain neighbour node with maximum mass proportion value using (8) \\
Measure the function (9) for obtaining optimal neighbouring node \\
Forward data packets through the obtained optimal neighbouring node
\end{tabular}




\begin{tabular}{|l|}
\hline End for \\
End for \\
End
\end{tabular}

Figure 6Greedy Local route search algorithm

Figure 6 shows the Greedy Local route search algorithm. Initially, the mass proportional value is obtained. With the obtained mass proportional value, the neighbour node with maximum mass proportion value is obtained. Followed by, the optimal neighbour node is measured based on the minimum energy and minimum distance between the sensor node and base station through which the data packet is transmitted. This in turn ensures minimum energy consumption for improving the network lifetime.

\section{EXPERIMENTAL SETTINGS}

In this section, the performance of GCR is evaluated through theoretical analysis and simulation study, including energy consumption, network lifetime with respect to different number of sensor nodes. The results of the metrics of GCR method is compared against the existing methods such as TPSO-CR (Two-tier Particle Swarm Optimization for Clustering and Routing) [1] and EDIT Energy Delay Index for Trade-off [2] respectively. The performance evaluations are based on the simulations of 70 sensor nodes that form a wireless sensor network over a rectangular space 1000 $\mathrm{m} * 1000 \mathrm{~m}$. Each node's radio propagation range is $300 \mathrm{~m}$ and data rate is $1 \mathrm{Mbps}$. Table 1 lists the set of input parameter and evaluates performance of GCR method via simulation that generates traffic for every $10 \mathrm{~m} / \mathrm{s}$.

The nodes are distributed in an area using Random Way point model for simulation, whereas the link layer provides the link between two sensor nodes and the design of link is multi direction. The base station collects the data packets of range $9-63$ and forwards the data packets to the base station with each data packet size differing from $100 \mathrm{~KB}$ to $512 \mathrm{~KB}$. The simulation time varies from 500 simulation seconds to 1500 simulation seconds.

Table 1 Simulation parameters

\begin{tabular}{|l|l|}
\hline Parameters & Values \\
\hline Area of sensing field & $1000 \mathrm{~m} * 1000 \mathrm{~m}$ \\
\hline Number of sensor nodes & 70 \\
\hline Number of data packets & $8,16,24,32,40,48,56$ \\
\hline Simulation time & $50 \mathrm{~s}$ \\
\hline Bandwidth & $1 \mathrm{Mbps}$ \\
\hline Propagation range & $300 \mathrm{~m}$ \\
\hline Simulation runs & 7 \\
\hline
\end{tabular}

\section{DisCUSSION}

To validate the analysis of the proposed Greedy Cluster-based Routing (GCR) in Wireless Sensor Network with existing TPSO-CR (Two-tier Particle Swarm Optimization for Clustering and Routing) [1] and EDIT Energy Delay Index for Trade-off [2], simulation results under NS2 are presented. The parameters of the GCR method are chosen as provided in the experiment section. 


\subsection{Scenario 1: Energy consumption}

Energy consumption between cluster head is measured using the energy consumed by a single sensor node with respect to the total sensor nodes in WSN. The mathematical formulation for energy consumption is as given below.

$$
E C=\sum_{i=1}^{n} \operatorname{Energy}(S) * \text { Total }\left(S_{i}\right)
$$

From (10), the energy consumption ' $E C$ ' between cluster head is obtained by the product of the energy for single node 'Energy $\left(S_{i}\right)$ ' and total sensor nodes 'Total $\left(S_{i}\right)$ ' in the network. The energy consumption is measured in terms of Joules.

Table 2 Performance evaluation of energy consumption

\begin{tabular}{|c|c|c|c|}
\hline \multirow{2}{*}{$\begin{array}{c}\text { Sensor node } \\
\text { density }\end{array}$} & \multicolumn{3}{|c|}{ Energy consumption (J) } \\
\cline { 2 - 4 } & GCR & TPSO-CR & EDIT \\
\hline 10 & 51 & 58 & 60 \\
\hline 20 & 62 & 75 & 80 \\
\hline 30 & 71 & 83 & 86 \\
\hline 40 & 83 & 91 & 95 \\
\hline 50 & 89 & 108 & 113 \\
\hline 60 & 98 & 115 & 120 \\
\hline 70 & 105 & 120 & 129 \\
\hline
\end{tabular}

As listed in table 2, GCR method measures the energy consumption between cluster head in WSN with respect to node density in the range of 10 to 70 sensor nodes. It is measured in terms of Joules (J). The energy consumption between cluster head in WSN using GCR method offers comparable values than the state-of-the-art methods.

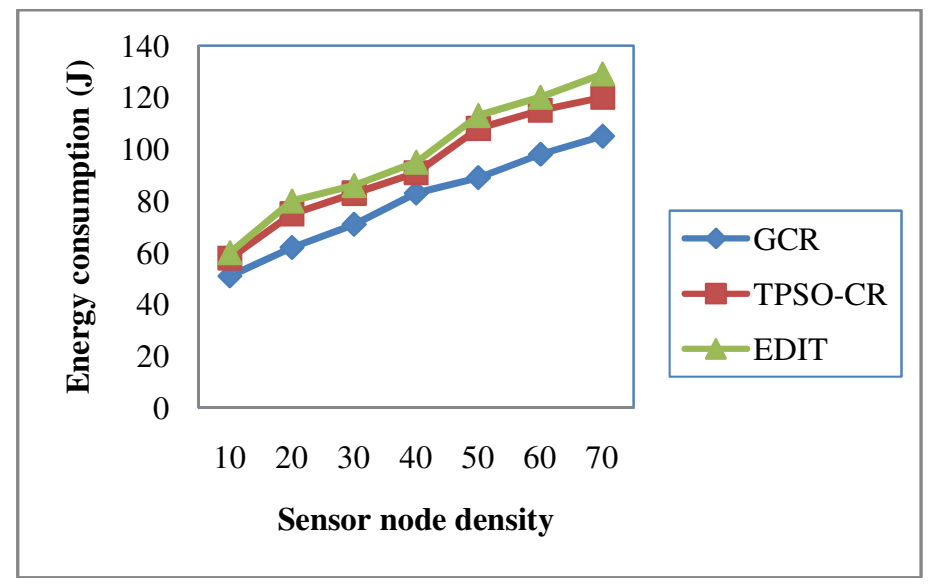

Figure 7 Measure of energy consumption with respect to sensor node density

Figure 7 presents the variation of energy consumption with respect to node density in wireless sensor network. All the results provided in figure 5 confirm that the proposed GCR method significantly outperforms the other two methods, TPSO-CR [1] and EDIT [2]. The energy consumption between cluster head is reduced in the GCR method using the cluster-based routing algorithm. With the application of cluster-based routing algorithm, arbitrary timer is applied to decide upon the cluster head node among the other sensor nodes in the network. The proposed 
GCR selects an optimal node through the local route search algorithm to forward data packet. The optimal nodes are selected based on nearer to destination node and has minimum energy. Therefore, GCR method obtains an optimal node for efficient packet transmission thereby enhancing the network lifetime with minimum energy consumption. The energy consumption is reduced by $16.31 \%$ compared to existing TPSO-CR [1]. As a result, energy consumption is reduced in the GCR method using the cluster based routing algorithm. Moreover, using the arbitrary timer, the sensor nodes participates in the cluster head selection process, uses only local information, further reduces the energy consumption between cluster head by $22.07 \%$ compared to existing EDIT [2].

\subsection{Scenario 2: Network lifetime}

The lifetime of the network is determined by the number of sensor nodes in the network in WSN. The network lifetime is expressed as given below.

$$
N L=\left(\frac{S_{\text {addressed }}}{\text { Total }_{S}}\right) * 100
$$

From (11), the network lifetime ' $N L$ ' is measured using the total number of sensor nodes 'Total ' in the network and the sensor node addressed ' $S_{\text {addressed }}$ ' in WSN. Higher the network lifetime, more efficient the method is said to be and is measured in terms of percentage (\%).

Table 3 Performance evaluation of network lifetime

\begin{tabular}{|c|c|c|c|}
\hline \multirow{2}{*}{$\begin{array}{c}\text { Sensor node } \\
\text { density }\end{array}$} & \multicolumn{3}{|c|}{ Network lifetime (\%) } \\
\cline { 2 - 4 } & GCR & TPSO-CR & EDIT \\
\hline 10 & 85.14 & 82.28 & 75.15 \\
\hline 20 & 89.16 & 86.76 & 79.20 \\
\hline 30 & 97.23 & 94.15 & 87.76 \\
\hline 40 & 82.14 & 79.81 & 70.23 \\
\hline 50 & 85.28 & 82.19 & 75.14 \\
\hline 60 & 88.18 & 85.66 & 78.32 \\
\hline 70 & 93.14 & 90.29 & 83.14 \\
\hline
\end{tabular}

The targeting results of network lifetime using GCR method with two state-of-the-art methods [1], [2] in table 3 presented for comparison based on the node density in wireless sensor network.

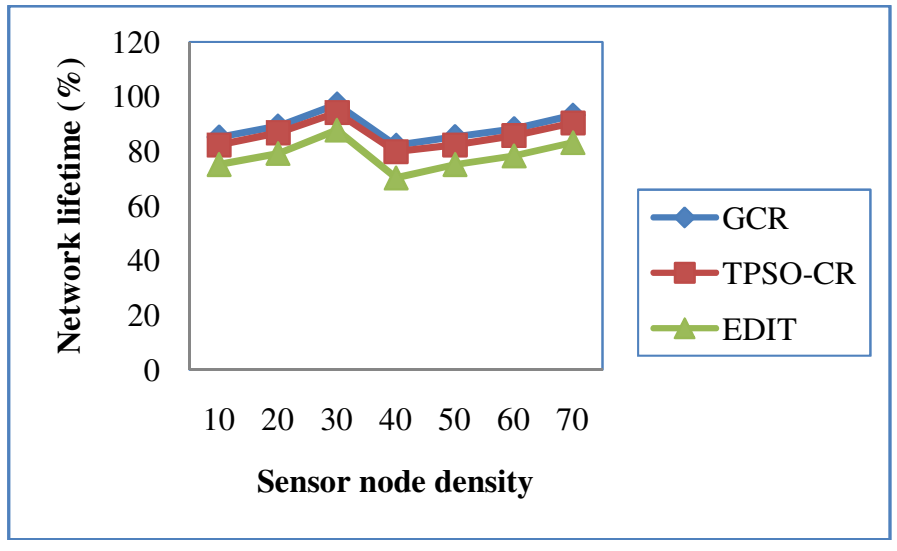

Figure 8 Measure of network lifetime with varied sensor nodes 
From figure 8, it is evident that the network lifetime is improved using the proposed GCR method than the existing methods. This is because; the GCR method uses cluster based routing algorithm uses arbitrary timer. In WSN, each sensor nodes starts with timer and its local information to contribute in the cluster head selection process. The node which has minimum energy consumption cost is selected during cluster head selection process. For transmitting a packet to destination, the neighbourhood node selection is essential. Therefore this process is obtained through applying mass proportion value. The GCR method uses only local information for choosing the cluster heads. Through this cluster head, the transmission is carried out effectively and reducing the control overhead. With the application of mass proportion value, neighbour node detection is performed in an efficient manner at different time intervals resulting in the improvement of network lifetime. At the same time, in GCR method, not only the mass proportion value is obtained for the sensor and neighbouring nodes but also the minimum distance between them is considered. This in turn increases the network lifetime using GCR method by $3.08 \%$ compared to TPSO-CR [1] and $11.56 \%$ compared to EDIT [2] respectively.

\subsection{Scenario: Throughput}

Throughput measures the rate of successful data packets delivery over a period of time interval in WSN. Therefore, throughput rate is the ratio of data packets sent by the source node and the data packets received by the destination node. It is measured in terms of percentage (\%) and is formulated as given below.

$$
T=\frac{D P_{r}}{D P_{s}} * 100
$$

From (12), the rate of throughput ' $T$ ' is measured using the data packets sent ' $D P_{S}$ ' and the data packets received ' $D P_{r}$ '. Higher the data packets being received, more efficient the method is said to be. To better understand the effectiveness of the proposed GCR method, extensive experimental results are reported in table 4 with respect to data packets sent.

Table 4 Performance evaluation of throughput

\begin{tabular}{|c|c|c|c|}
\hline \multirow{2}{*}{ Data packets } & \multicolumn{3}{|c|}{ Throughput } \\
\cline { 2 - 4 } & GCR & TPSO-CR & EDIT \\
\hline 8 & 85.28 & 81.23 & 73.21 \\
\hline 16 & 87.14 & 83.19 & 76.29 \\
\hline 24 & 89.38 & 85.15 & 77.29 \\
\hline 32 & 92.23 & 88.20 & 81.32 \\
\hline 40 & 84.19 & 80.20 & 73.78 \\
\hline 48 & 87.32 & 83.18 & 78.14 \\
\hline 56 & 90.14 & 86.67 & 80.29 \\
\hline
\end{tabular}

NS2 simulator is used to experiment throughput rate by analyzing the result using table and graph values. Results are presented for different number of data packets and the results reported here confirm that with the increase in the number of data packets, the rate of throughput also gets increased. Though, the increase is not linear. This is due to the sensor node movements at different time periods. 


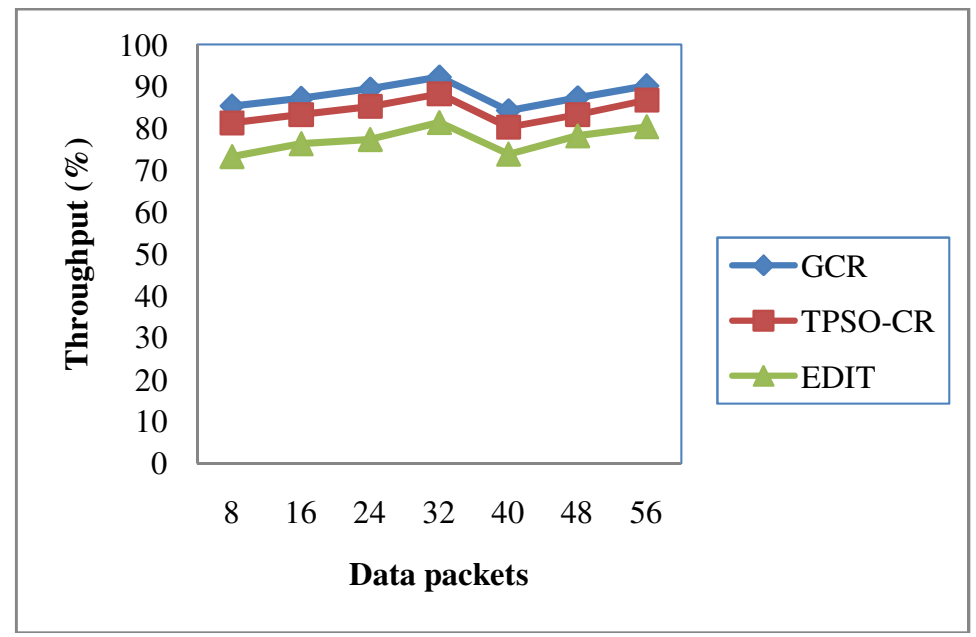

Figure 9 Measure of throughput

Figure 9 shows the rate of throughput with respect to different number of data packets with size in the range of $100 \mathrm{~KB}$ to $512 \mathrm{~KB}$ in wireless sensor network considered for experimental purpose. As shown in figure, the proposed GCR method performs relatively well when compared to two other existing methods TPSO-CR [1] and EDIT [2]. This is because; the rate of throughput is improved in the GCR method by applying Greedy strategy through local route search model. By applying the Greedy Local Route Search algorithm, the neighbouring node is selected based on the maximum mass proportional value. With the higher mass proportional value of the node, a function with sensor nodes consuming minimum energy and minimum distance between the nodes are selected for data packet forwarding. This helps in increasing the rate of throughput by $4.53 \%$ compared to TPSO-CR [1]. With obtained optimal neighbour node using the function in GCR method, the rate of throughput is improved in GCR method by $12.25 \%$ compared to EDIT [2] respectively.

\section{CONCLUSION}

In this paper, Greedy Cluster-based Routing (GCR) method is developed based on the Clusterbased Routing Protocol and Mass Proportion-based Neighbour Node selection with Greedy strategy for wireless sensor network. The GCR method minimizes the energy consumption between cluster head and therefore improves the network lifetime. The GCR method uses clusterbased routing algorithm in a dynamic manner, it reduces the energy consumption between cluster head in WSN through arbitrary timer. As a result, the proposed cluster-based routing algorithm selects the cluster head in an efficient manner and helps in minimizing the energy consumption between cluster head and therefore improving the efficiency of the system and the overall network. By applying the Mass Proportion-based Neighbour Node selection with the maximum proportion value, improves the network lifetime in WSN. Finally, the greedy strategy based local route search algorithm is considered in a significant manner and therefore improving the rate of throughput. Different sensor nodes with varied packet sizes on WSN using GCR method analyze the routing efficiency to significantly improve the energy savings and therefore the network lifetime in WSN. A series of simulation results are performed to test the energy consumption, network lifetime and throughput rate. The simulation results show that GCR method offers better performance with an improvement of network lifetime by $7.32 \%$ and improves the throughput rate by $8.39 \%$ compared to TPSO-CR [1] and EDIT [2] respectively. 
International Journal of Computer Science \& Information Technology (IJCSIT) Vol 9, No 2, April 2017

\section{REFERENCES}

[1] Riham S.Y. Elhabyan, Mustapha C.E. Yagoub," Two-tier particle swarm optimization protocol for clustering and routing in wireless sensor network", Journal of Network and Computer Applications, Elsevier, Vol. 52, June 2015, pp. 116-128.

[2] Ankit Thakkar, Ketan Kotecha," Cluster Head Election for Energy and Delay Constraint Applications of Wireless Sensor Network", IEEE Sensors Journals, Vol. 14, No.8, Aug 2014, pp.2658-2664

[3] Padmalaya Nayak, and D. Anurag," A Fuzzy Logic based Clustering Algorithm for WSN to extend the Network Lifetime", IEEE Sensors Journals, Vol. 16, No.1, 2016, pp.137-144.

[4] Ankit Thakkara, Ketan Kotecha," A new Bollinger Band based energy efficient routing for clustered wireless sensor network", Applied Soft Computing, Elsevier, Vol.32, July 2015, pp. 144-153.

[5] Sabet Maryam, Naji Hamid Reza, "A decentralized energy efficient hierarchical cluster-based routing algorithm for wireless sensor networks", AEU -International Journal of Electronics and Communications, Elsevier, Vol. 69, No.5, May 2015, pp.790-799.

[6] Yongsheng Ding, Rui Chen, and Kuangrong Hao," A Rule-Driven Multi-Path Routing Algorithm with Dynamic Immune Clustering for Event-Driven Wireless Sensor Networks", Vol. 203, Aug 2016, pp. 139-149

[7] Tao Dua, Shouning Qu, Fangai Liu, Qin Wanga,” An energy efficiency semi-static routing algorithm for WSNs based on HAC clustering method", Information Fusion, Elsevier, Vol. 21, Jan 2015, pp.1829.

[8] Ado Adamou ABBA ARI, Blaise Omer YENKE, Nabila LABRAOUI, Irepran DAMAKOA, Abdelhak GUEROUI," A power efficient cluster-based routing algorithm for wireless sensor networks : Honeybees Swarm Intelligence based approach", Journal of Network and Computer Applications, Elsevier, Vol. 69, July 2016, pp.77-97.

[9] Zeynab Molay Zahedi, Reza Akbari, Mohammad Shokouhifar, Farshad Safaei, Ali Jalali," Swarm intelligence based fuzzy routing protocol for clustered wireless sensor networks", Expert Systems with Applications, Elsevier, Vol.55, August 2016, pp.313-328.

[10] Pratyay Kuila n, PrasantaK.Jana," Energy efficient clustering and routing algorithms for wireless sensor networks: Particle swarm optimization approach", Engineering Applications of Artificial Intelligence, Elsevier, Vol.33, August 2014, pp.127-140.

[11] Md Azharuddin, Pratyay Kuila, Prasanta K. Jana," Energy efficient fault tolerant clustering and routing algorithms for wireless sensor networks", Computers and Electrical Engineering, Elsevier, Vol.41, January 2015, pp.177-190.

[12] Hui Lin and Halit Üster," Exact and Heuristic Algorithms for Data-Gathering Cluster-Based Wireless Sensor Network Design Problem", IEEE/ACM Transactions on Networking, Vol. 22, no.3, June 2014, pp.903-916.

[13] Hai Lin, Lusheng Wang and Ruoshan Kong," Energy Efficient Clustering Protocol for Large-Scale Sensor Networks", IEEE Sensors Journal, Vol. 15, no. 12, Dec 2015, pp.7150-7160

[14] Ashfaq Ahmad, Nadeem Javaid, Zahoor Ali Khan, Umar Qasim, and Turki Ali Alghamdi," (ACH)2: Routing Scheme to Maximize Lifetime and Throughput of Wireless Sensor Networks", IEEE Sensors Journal ,Vol. 14, no. 10, Oct. 2014, pp.3516-3532

[15] Farhan Aadil, Khalid Bashir Bajwa, Salabat Khan, Nadeem Majeed Chaudary, Adeel Akram," CACONET: Ant Colony Optimization (ACO) Based Clustering Algorithm for VANET", PLOS ONE journal, Vol. 11, no. 5, pp.

[16] Suraj Sharma, Sanjay Kumar Jena," Cluster based Multipath Routing Protocol for Wireless Sensor Networks", ACM SIGCOMM Computer Communication Review, Vol. 45, no. 2, April 2015, pp. 1420.

[17] Sohail Jabbar, Abid Ali Minhas, Anand Paul, Seungmin Rho," Multilayer cluster designing algorithm for lifetime improvement of wireless sensor networks", Journal of Supercomputing, Springer, Vol.70, no. 1 , Oct 2014, pp. 104-132.

[18] M. Mehdi Afsar," Effective Data Aggregation Scheme for Large-scale Wireless Sensor Networks", IEEE 27th Canadian Conference on Electrical and Computer Engineering, May 2014.

[19] Xuemei Sun, Bo Yan, Xinzhong Zhang, Chuitian Rong," An Integrated Intrusion Detection Model of Cluster-Based Wireless Sensor Network", PLOS ONE journal, Vol.10, no.10, October 8, 2015.

[20] Vahid Ayatollahitafti, Md Asri Ngadi, Johan bin Mohamad Sharif, Mohammed Abdullahi," An Efficient Next Hop Selection Algorithm for Multi-Hop Body Area Networks", PLOS ONE journal, Vol.11, no.1, Jan 152016. 


\section{Authors}

M. PARTHASARATHI, received the B.Sc.,(Physics), M.Sc (CS) fromBharathidasan University, Trichy, in 1992 - 1998 and ME in computer science Engineering in Anna University, Coimbatore in 2008. Currently,He is pursuing the Ph.D. degree in Computer Science in MS university,Tirunelveli. His research interests wireless networks. He has more than 17 years teaching experience in various colleges. Now currently he is working in KSR college of Arts and Science, Tiruchengode from 2005 to till date.

Dr. KARTHIKEYANI V was born in Tamil Nadu, India in 1972. She is working as an Assistant Professor in Department of Computer Science at Govt. Arts College, Rasipuram, Tamilnadu, India. She was awarded Doctoral degree from Periyar Universtiy, Salem, Tamilnadu, India. She has published 33 National and International Journals and presented several papers in International and National Conferences. She has 21 years of teaching

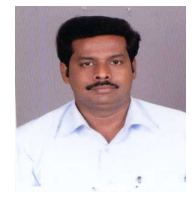
experience. Her areas of interests are Image Processing, Computer Graphics, Multimedia, Data Mining and Web Mining. She is a life member in Computer Society of India (CSI), ISTE (Indian Society for Technical Education), ACM-CSTA (Computer Science Teacher Association). 